\title{
Synthesis and Application of Ag NPs@graphene Oxide Nanocomposite as an Electrochemical Sensor for Sweat Lactate Monitoring in Sports Medicine
}

\author{
Wenhong Wang \\ Department of Physical Education, Jinling Institute of Technology, Nanjing, 211169, China \\ E-mail: wwh@jit.edu.cn
}

Received: 28 August 2021 / Accepted: 9 October 2021 / Published: 10 November 2021

\begin{abstract}
In this study, a sensitive electrochemical sensor for detection of lactate was proposed. The sensor was developed based on GCE substrate coated by Ag NPs@GO/GCE. The morphological and structural properties of Ag NPs@GO/GCE were characterized using FESEM and XRD analysis, respectively. FESEM results confirmed a uniform distributed Ag NPs in GO nanocomposites. The sensor performance of the device was examined by amperometry and cyclic voltammetry. The electrochemical sensor exhibited a linear response to the lactate in phosphate buffer solution with a liner range and sensitivity of 10 to $600 \mu \mathrm{M}$ and $16.5 \mu \mathrm{Acm}^{-2} \mathrm{mM}^{-1}$, respectively. The concentration of lactate estimated by this sensor in real sweat samples was very close to the amount of injection which can be considered as a sensor for lactate measurement in sweat samples.
\end{abstract}

Keywords: Electrochemical sensor; lactate; Ag NPs@graphene oxide nancomposites; Cyclic voltammetry

\section{FULL TEXT}

(C) 2021 The Authors. Published by ESG (www.electrochemsci.org). This article is an open access article distributed under the terms and conditions of the Creative Commons Attribution license (http://creativecommons.org/licenses/by/4.0/). 